\title{
LAS ENFERMEDADES CRÓNICAS NO TRANSMISIBLES, UN RETO POR ENFRENTAR
}

\author{
CHRONIC NON-COMMUNICABLE DISEASES, A CHALLENGE TO FACE \\ Germán Málaga1,2,a,b \\ Las enfermedades crónicas no transmisibles (ECNT) promueven, desde 1996, cambios sustantivos en el \\ patrón de enfermedad en el Perú. Su progresión ha sido tan eficiente que prevalecen en más de la mitad \\ de departamentos y en la totalidad de los de la costa, que es la zona más poblada del país ${ }^{(1)}$. Dentro del \\ heterogéneo grupo que constituyen las ECNT, las enfermedades cardiovasculares fueron las que más \\ muertes produjeron en el mundo, seguidas de cáncer, enfermedades pulmonares obstructivas crónicas \\ (EPOC) y diabetes, siendo responsables del $80 \%$ de mortalidad atribuible a las ECNT ${ }^{(2)}$, situación que \\ es semejante en nuestro medio, pues, en el 2010 fueron causantes de más de $2 / 3$ de todas las muertes \\ ocurridas en el Perú (3).
}

En cuanto al impacto de estas enfermedades, un análisis del 2010, reveló que del cálculo de años perdidos por muertes prematuras y años vividos con discapacidad (DALYs) el $54 \%$ correspondió a las ECNT ${ }^{(4)}$. No tenemos información reciente en Perú, pero según un estudio de carga de enfermedad, en el año 2004 se perdieron más de 3 millones de años de vida saludables como consecuencia de ECNT, lo que representó el $60 \%$ del total registrado ${ }^{(5)}$.

Estas enfermedades concitan preocupación por lo difícil que resulta su control, por ejemplo en hipertensión arterial, en el 2010 aproximadamente 78 millones de estadounidenses eran portadores, y solo el $50 \%$ de ellos tenían un buen control ${ }^{(6)}$, situación particularmente interesante, pues un gran porcentaje de pacientes con hipertensión se atienden en la red de atención primaria. Según información del INEI, existirian en el Perú más de 2000000 de pacientes portadores de hipertensión arterial, y si solo la mitad de ellos sabe que es portador de la enfermedad, es decir pacientes con diagnóstico conocido y que acuden a hospitales para su control, solo $40 \%$ tienen adecuada adherencia a la medicación prescrita ${ }^{(7)}$, tenemos que el futuro de la enfermedad, en cuanto a complicaciones que pudieran ser prevenibles, se avizora como problemático, con lo que las consecuencias para los sistemas de salud global, y para el Perú en particular, será tremendo.

Ante este panorama, ¿qué pasa con un paciente portador de una ECNT en el Perú?, probablemente, una respuesta la obtengamos de investigaciones aún no publicadas, por ejemplo, en pacientes que sufrieron su primer accidente cerebro vascular, menos de la mitad de ellos regresa a su control al mes del evento, solo un $30 \%$ completa un programa de terapia física y la mortalidad al año del evento es $20 \%$. Esta situación tan poco estimulante, pero a su vez gráfica, puede ser atribuida a una serie de infortunios, pero fundamentalmente revela falta de concreción de la asociación que debe tener el médico con el paciente y la falta de un sistema de seguimiento para estos pacientes de alto riesgo.

Lo descrito nos lleva afirmar que aún estamos viviendo en un periodo de "transición epidemiológica", la que no solo denota una coexistencia entre enfermedades crónicas no transmisibles y enfermedades transmisibles, sino que nos expone a una simbiosis, pues se describe cada vez con más frecuencia la interrelación entre dos enfermedades como son la tuberculosis y diabetes ${ }^{\left({ }^{8}\right)}$, cuyo manejo clínico y control a nivel de salud pública se convierte en un nuevo reto para cualquier sistema de salud como lo

\footnotetext{
CRONICAS Centro de Excelencia en Enfermedades Crónicas, Universidad Peruana Cayetano Heredia. Lima, Perú.

Hospital Nacional Cayetano Heredia. Lima, Perú.

Editor invitado; ${ }^{\mathrm{b}}$ Médico internista

Recibido: 15-12-13 Aprobado: 08-01-14

Citar como: : Málaga G. Las enfermedades crónicas no transmisibles, un reto por enfrentar [editorial]. Rev Peru Med Exp Salud Publica. 2014;31(1):6-8. .
} 
mencionan los doctores César Ugarte y David Moore en un artículo publicado en este número.

Algunas características hacen diferentes a estas enfermedades y es que, como su nombre lo indica, son crónicas, es decir, no se curan, lo que obliga a que la persona tenga que convivir, en la mayor parte de los casos, de manera permanentemente con ellas y, de otro lado, su emergencia es el resultado de una serie de circunstancias irreversibles y de alguna manera deseables, como la mejora en el nivel económico de los pobladores, pero, a costa de urbanización y migración a las ciudades, con la adopción de estilos de vida no saludables en alimentación y en deficiente actividad física, lo que conlleva a que, para su control, se requiera de un cambio en la concepción y la incorporación de cambios en el estilo de vida.

Desde la perspectiva del personal de salud, el advenimiento de las ECNT, requieren un cambio de paradigmas, como se señala en un artículo del simposio, escrito por el Dr. Gordon Guyatt ${ }^{(9)}$, prolífico investigador afiliado a McMaster University, quien entre sus méritos tiene haber desarrollado y acuñado el término de "Medicina Basada en la Evidencia", quien discute los problemas relacionados con la interpretación de la investigación y el hecho de que las intervenciones terapéuticas en las ECNT, no sean curativas, sino que solo reducen el riesgo de que ocurra una complicación, o un evento, es decir, nos obliga a que tengamos que lidiar con la incertidumbre. Esta situación dificulta aspectos relacionados con la comunicación y transmisión de información al paciente, de modo que la posibilidad de compartir la decisión terapéutica y hacer que mediante este proceso, el paciente pase a formar parte del equipo terapéutico, se sienta confortado o con menos estrés con relación al tratamiento ${ }^{(10)}$, con las consiguientes mejoras en la adherencia al tratamiento (situación crítica para el control de estas enfermedades) o lograr que el paciente tenga interés en educarse con relación a su enfermedad y se empodere de la misma, con los beneficios demostrados en cuanto a control se refiere ${ }^{(11)}$.

Otro grupo de ECNT, lo constituyen las enfermedades mentales, y como revisa en el presente número el Dr. Jaime Miranda et. al. investigador que lidera el Centro de Excelencia en enfermedades CRONICAS de la UPCH ${ }^{(12)}$, la disrupción que originan las ECNT en la vida de las personas, hace que se establezca una "relación simbiótica"; así las ECNT promueven su aparición, en especial depresión, la cual impide que el tratamiento de las mismas sea eficiente, afectando la adherencia al tratamiento o promoviendo la inactividad física. Esto afectará aun más la calidad de vida, la satisfacción con relación a su enfermedad, sumando no solo carga de enfermedad, sino, morbilidad y mortalidad, pues el riesgo de mortalidad en diabéticos con depresión, es 2,3 veces mayor ${ }^{(12)}$. Si a esto le sumamos el hecho de que ya existe una autopercepción de parte de los pacientes de necesidades no satisfechas con relación a atención a salud mental, la explosión de las ECNT solo agravara esa situación (13).

Una ECNT importante en cuanto a morbilidad, mortalidad y carga de enfermedad, lo constituyen las EPOC, en este número el Dr. William Checkley ${ }^{(14)}$, investigador de Johns Hopkins University y de CRONICAS, hace una revisión de lo que representaría la EPOC, producido como consecuencia del uso de combustibles orgánicos, afectando a las poblaciones rurales económicamente desfavorecidas de la costa y del ande. Se debe tener en cuenta que la polución intradomiciliaria afecta a todo el núcleo familiar, y enferma no solo a la vía respiratoria, sino que desencadena una reacción inflamatoria crónica de consecuencias imprevisibles y aún por definir, precisamente el Dr. Checkley viene realizando estudios relacionados en Puno y Tumbes, que nos darán luces de la real magnitud del problema.

La situación descrita, es decir, varias enfermedades concurriendo, la necesidad de varios especialistas interviniendo en el cuidado de la salud de una misma persona, con la consecuente polifarmacia, estrés y alteración en la calidad de vida, ha llamado la atención y originado una amplia discusión en varios centros académicos, uno de los líderes globales de esta discusión, es el Dr. Victor Montori (15), profesor de Medicina y endocrinólogo de la Clínica Mayo. Esta discusión ha generado una corriente de pensamiento en torno a la denominada "medicina mínimamente intrusiva" cuyo punto central gira en torno a la aplicación no solo de la mejor evidencia, sino la más eficiente, y que tenga en cuenta los valores y preferencias del paciente portador de una o varias ENT, buscando un balance entre lo mínimamente intrusivo frente a lo realmente eficiente. En este artículo se hace un análisis de lo que representa la medicina centrada en la enfermedad o en el médico frente a lo que sería la medicina centrada en el paciente y lo que representa "el trabajo que significa ser paciente".

A los problemas y complejidades descritos, un problema agregado tiene que ver con que a pesar de la ingente cantidad de investigación y por tanto, de evidencia producida, existe gran variabilidad en la práctica clínica lo que ocasiona que esa evidencia no sea aplicada, afectando de manera directa a los pacientes. En un intento por uniformizar la práctica clínica es que diversas sociedades científicas, académicas y gobiernos vienen implementando guías de práctica clínica, sin embargo, su impacto se reporta en general como exiguo, básicamente por ser documentos extensos, contra lo que su nombre 
indicaría, poco prácticos, por lo que su diseminación e implementación es un tema pendiente a todo nivel ${ }^{(16)}$. El Dr. Per O. Vandvik, del Inlnandet Hospital Trust, Gjøvik, Noruega y su grupo de investigación, vienen desarrollando un sistema que, como su nombre lo indica, pareciera ser "Magic", pues permite en un software tener acceso a la historia electrónica de los pacientes y del CIE-10 digitado, obtener información de cómo verificar el diagnóstico, estratificar la enfermedad y obtener información sobre cuál es la mejor evidencia recomendada, evaluada de acuerdo a la metodología GRADE (17,18). Esta iniciativa viene teniendo gran acogida entre los diversos actores productores de GPC, como una herramienta que permite implementar las GPC de manera sencilla y, sobre todo, permitir su diseminación de manera efectiva.

Finalmente, vemos que los esfuerzos para mejorar su control y evitar las terribles consecuencias de estas enfermedades aún son aisladas, por un lado, el sector privado en un afán por reducir costos viene implementando programas donde se facilita la administración de medicamentos a los pacientes y tibiamente se implementan programas educativos, pero sin la perspectiva de hacer de ellos agentes de cambio por medio de la emancipación en sus enfermedades, y que comanden el control de las mismas. Por el lado del sector publico, aún no se tienen concreciones, más allá que establecer días dedicados a algunas de estas enfermedades, la implementación y ejecución de programas como el Plan Esperanza (enfocado por el momento en el desarrollo de actividades en cáncer), campañas de salud aisladas y enmarcadas en esfuerzos internacionales, aún no se ha logrado concreción de un programa de prevención y control para estas enfermedades, que por lo demás, es difícil, dada la heterogeneidad de las mismas y los diferentes requerimientos de cada una de ellas. Así que, ante la pregunta de si estamos preparados ante la llegada y ascenso de las enfermedades crónicas no transmisibles, la respuesta cruda y sincera, es: no estamos preparados, individualmente ni como sistema de salud y tenemos un reto muy difícil por afrontar.

\section{REFERENCIAS BIBLIOGRÁFICAS}

1. Huicho L, Trelles M, Gonzales F, Mendoza W, MirandaJ. Mortality profiles in a country facing epidemiological transition: an analysis of registered data. BMC Public Health. 2009;9:47. doi: 10.1186/1471-2458-9-47.

2. Lozano R, Naghavi M, Foreman K, Lim S, Shibuya K, Aboyans V, et.al. Global and regional mortality from 235 causes of death for 20 age groups in 1990 and 2010: a systematic analysis for the Global Burden of Disease Study 2010. Lancet. 2012;380(9859):2095-128. doi: $10.1016 /$ S0140-6736(12)61728-0.

3. World Health Organization. Peru - Noncommunicable diseases [Internet]. Geneva: WHO; 2011 [updated 2011; citado el 27 de diciembre del 2013]. Disponible en: http:// www.who.int/nmh/countries/per_en.pdf

4. Murray CJ, Vos T, Lozano R, Naghavi M, Flaxman AD, Michaud C, et al. Disability-adjusted life years (DALYs) for 291 diseases and injuries in 21 regions, 1990-2010: a systematic analysis for the Global Burden of Disease Study 2010. Lancet. 2012;380(9859):2197-223. doi: 10.1016/S0140-6736(12)61689-4.

5. Perú, Ministerio de Salud. Estudio de Carga de Enfermedad en el Perú-2004. MINSA. Lima, 2006 [citado el 2 diciembre 2013] Disponible en: http://www.dge. gob.pe/pub_asis.php?_pagi_pg=2

6. Go AS, Mozaffarian D, Roger VL, Benjamin EJ, Berry JD, Blaha MJ, et al. Heart disease and stroke statistics--2014 up- date: a report from the American Heart Association Circulation. Circulation. 2014;129(3):e28-e292. doi: 10.1161/01. cir.0000441139.02102.80.

7. Carhuallanqui R, Diestra-Cabrera G, Tang-Herrera J, Málaga G. Adherencia al tratamiento farmacológico en pacientes hipertensos atendidos en un hospital general. Rev Med Hered. 2010;21(4):197-201.

8. Ugarte-Gil C, Moore DAJ. Comorbilidad de tuberculosis y diabetes: problema aún sin resolver. Rev Peru Med Exp Salud Publica. 2014;31(1):137-42.

9. Guyatt GH, Málaga G. Dificultades en la interpretación de los resultados de la investigación biomédica relacionada con el manejo de pacientes con enfermedades crónicas no transmisibles. Rev Peru Med Exp Salud Publica. 2014;31(1):127-30.

10. Charles C, Gafni A WT. Decision-making in the physician-patient encounter: revisiting the shared treatment decision-making model. Soc Sci Med. 1999;49(5):651-61.

11. Montori VM, Gafni A, Charles C. A shared treatment decision-making approach between patients with chronic conditions and their clinicians: the case of diabetes. Health Expect. 2006;9(1):25-36.

12. Diez-Canseco F, Ipince $A$, Toyama $M$, Benate-Galvez Y, Galán-Rodas E, MedinaVerástegui JC, et al. Atendiendo la salud mental de las personas con enfermedades crónicas no transmisibles en el Perú: retos y oportunidades para la integración de cuidados en el primer nivel de atención. Rev Peru Med Exp Salud Publica. 2014;31(1):131-6.

13. Gonzalez JS, Fisher L, Polonsky WH. Depression in diabetes: have we been missing something important? Diabetes Care. 2011;34(1):236-9. doi: 10.2337/ dc10-1970.

14. Lopez M, Mongilardi N, Checkley W. Enfermedad pulmonar obstructiva crónica por exposición al humo de biomasa. Rev Peru Med Exp Salud Publica. 2014;31(1):94-9.

15. Zeballos-Palacios C, Morey-Vargas OL, Brito JP, Montori VM. Toma de decisiones compartidas y medicina mínimamente impertinente en el manejo de las enfermedades crónicas. Rev Peru Med Exp Salud Publica. 2014;31(1):111-7.

16. von Dincklage JJ, Ball D, Silvestri GA. A review of clinical practice guidelines for lung cancer. J Thorac Dis. 2013;5(Suppl 5):S607-S622.

17. Olav Vandvik P, Fog Heen A, Brandt L. Una nueva generación de guías de práctica clínica confiables a través de MAGIC. Rev Peru Med Exp Salud Publica. 2014;31(1):118-26.

18. Atkins D, Eccles M, Flottorp S, Guyatt GH, Henry D, Hill S, et al. Systems for grading the quality of evidence and the strength of recommendations I: critical appraisal of existing approaches The GRADE Working Group. BMC Health Serv Res. 2004;4(1):38. 